\title{
VEGETACIÓN ACUÁTICA Y HELOFÍTICA DE LA DEPRESIÓN DE PADUL (GRANADA)
}

\author{
Francisco PÉREZ RAYA y Juan Manuel LÓPEZ NIETO
}

\begin{abstract}
RESUMEN. Se presenta un estudio de la vegetación acuática y helofítica de la Depresión de Padul. Se reconocen seis comunidades acuáticas, una terofítica y cinco helofíticas pertenecientes a las clases fitosociológicas: Lemnetea, Ceratophylletea, Charetea Potametea, Isoeto-Nanojuncetea y Phragmitetea.
\end{abstract}

Palabras clave. Vegetación hidrofítica. Granada.

ABSTRACT. In the present work a study of aquatic and helophytic vegetation in the Depresion de Padul is presented. Six water-plant communities, one annual and five helophytic belonging to the fitosociological classes: Lemnetea, Ceratophylletea, Charetea, Potametea, Isoeto-Nanojuncetea and Phragmitetea are recognized.

Key words. Hidrophytic vegetation. Granada. Spain.

\section{INTRODUCCIÓN}

La Depresión de Padul, con una extensión aproximada de $15 \mathrm{~km}^{2}$ se localiza en el límite occidental de Sierra Nevada y ocupa la zona más septentrional del Valle de Lecrín. Aparece limitada por las estribaciones de Sierra Nevada -Silleta del Manar- al N y NE, pequeñas lomas que se elevan hacia la Sierra de Albuñuelas al S y SW y cerrada al SE por los materiales depositados por el río Dúrcal.

Desde el punto de vista geológico, la Depresión de Padul es una fosa tectónica subsidente, limitada por fracturas de dirección NW-SE; aparece colmatada por materiales postorogénicos de naturaleza detrítica (limos y arcillas) que alternan con formaciones de tipo lacustre (arenas y turba). Constituye el drenaje natural de una amplia cuenca hidrológica, que al quedar cerrada por el cono de deyección del río Dúrcal, se instauró a finales del Plioceno un medio lagunar (cuenca de tipo endorreico) que condicionó el desarrollo posterior de una turbera.

Esta laguna, una de las más extensas de Andalucía Oriental, fue drenada 
artificialmente por motivos agrícolas y sanitarios a finales del siglo XVIII. El drenaje se realizó mediante una serie de canales que además de encauzar las aguas que afloran superficialmente, recogen también las descargas subterráneas definidas y difusas. Actualmente los principales aprovechamientos que se realizan en la zona son la extracción de turba y los cultivos de regadío.

Sin embargo, los canales no consiguieron desecar por completo la laguna, quedando determinados reductos lagunares, sobre todo en los sectores donde se extrae turba. Bordeando estas pequeñas lagunas, aparecen extensas zonas que se presentan más o menos encharcadas casi todo el año, observándose en los últimos 25 años un considerable aumento de la superficie pantanosa (que supone unos 900.000 $\mathrm{m}^{2}$ en el año 1985).

Biogeográficamente y de acuerdo con Rivas-Martínez et al.(1987), la Depresión de Padul queda incluida en el subsector Alfacarino-Granatense (sector MalacitanoAlmijarense, provincia Bética), correspondiendo su vegetación potencial climatófila a la serie mesomediterránea bética, marianense y araceno-pacense seco-subhúmeda basófila de la encina: Paeonio-Querceto rotundifoliae Sigmetum (Rivas-Martínez, 1987).

Los datos climáticos proporcionados por Elías y Ruiz Beltrán (1977), para la estación de Padul, son los siguientes: T: $16,0^{\circ} \mathrm{C} ; \mathrm{M}: 14,3^{\circ} \mathrm{C} ; \mathrm{m}: 3,7^{\circ} \mathrm{C} ; \mathrm{I}_{\mathrm{t}}: 340 ; \mathrm{P}$ : $474 \mathrm{~mm}$, lo que nos permite ubicar bioclimáticamente a la Depresión de Padul en el horizonte inferior del piso mesomediterráneo, presentando un ombroclima seco, caracterizado por un período de sequía estival prolongada y un invierno de tipo templado, en que ningún mes la temperatura media es inferior a $7,5^{\circ} \mathrm{C}$.

\section{DESCRIPCIÓN DE LAS COMUNIDADES}

A pesar de las constantes agresiones del hombre sobre la Depresión de Padul y en concreto sobre su sistema lagunar, aún hoy día se conserva una interesante vegetación acuática y helofítica en la zona, constituyendo la mejor representación de los sistemas palustres en la provincia de Granada. En el presente trabajo describimos las comunidades acuáticas y helofíticas que hemos reconocido en la Depresión de Padul, constituyendo buena parte de ellas la primera referencia en la zona, y ampliándose de forma considerable la corología de otras.

\section{I.- LEMENETEA MINORIS W.Koch \& R.Tx. in R.Tx. 1955}

Clase de distribución cosmopolita que agrupa a la vegetación dulceacuícola no enraizada constituida por acro y mesopleustófitos de pequeña talla. Se encuentra representada por el orden Lemnetalia minoris y la alianza Lemnion gibbae. 


\section{1.- Lemnetum gibbae (W.Koch 1954) Miyawaki \& R.Tx. 1960}

Asociación constituida por pequeños acropleustófitos (lémnidos) que forman parte del epipleon de aguas permanentes, remansadas, eutrofizadas y ricas en iones. Se presenta en forma de poblaciones prácticamente monoespecíficas de Lemna gibba (tabla 1), que llegan a cubrir por completo la superficie del agua. Debido al elevado grado de contaminación de las aguas en que se desarrolla, la presencia de Lemna minor es muy puntual, ya que soporta menos la eutrofización. Destaca la presencia como compañera en un inventario de Zannichellia peltata, que según Rivas-Martínez et al. (1980) caracteriza a la clase RUPPIETEA J.Tx. 1960, mientras que en la zona se presenta en aguas dulces muy eutrofizadas.

TABLA 1

Lemnetum gibbae (W.Koch 1954) Miyawaki \& R.Tx. 1960

(Lemnion, Lemnetalia, Lemnetea)

\begin{tabular}{lcr}
\hline № de inventario & 1 & 2 \\
Area $\left(\mathrm{m}^{2}\right)$ & 2 & 6 \\
Cobertura (\%) & 100 & 90 \\
№ de especies & 2 & 2 \\
& \multicolumn{2}{c}{} \\
Caract. de asociación y unidades superiores \\
Lemna gibba & 5.5 & 4.4 \\
Lemna minor & + &. \\
& & \\
Compañeras & & \\
Zannichellia peltata &. & 3.3
\end{tabular}

Localidades: 1.- Acequia del Ventano (VF4696). 2.- Turbera de Aguadero (VF4697).

\section{II.- CERATOPHYLLETEA Den Hartog \& Segal 1964}

Vegetación constituida por pleustófitos sumergidos (mesopleustófitos), cuyos órganos asimiladores muestran las hojas finamente divididas en lacinias, y carecen de hojas flotantes en superficie; en general son comunidades oligofíticas. Representada por el orden Ceratophylletalia, propio de aguas eútrofas, y la alianza Ceratophyllion.

2.- Comunidad de Ceratophyllum demersum $\mathrm{L}$.

En diversas charcas y zonas de aguas remansadas de las acequias del territorio, hemos podido reconocer una comunidad de Ceratophyllum demersum (tabla 2), 
incluible sin dificultad en la jerarquía sintaxonómica mencionada. Suele presentar un desarrollo considerable, formando masas densas que con frecuencia se sitúan entre helófitos de talla media o elevada, como Typha dominguensis, Sparganium erectum, etc., que les sirven de protección. Muy escasa en Andalucía, se trata de un tipo de vegetación claramente en regresión, por lo que resulta de gran importancia la buena representación que mantiene en el territorio.

\section{TABLA 2}

Comunidad de Ceratophyllum demersum L. (Ceratophyllion, Ceratophylletalia, Ceratophylletea)

$\begin{array}{lrrrrr}\text { № de inventario } & 1 & 2 & 3 & 4 & 5 \\ \text { Area }\left(\mathrm{m}^{2}\right) & 5 & 9 & 10 & 2 & 10 \\ \text { Cobertura }(\%) & 100 & 90 & 90 & 80 & 80 \\ \text { № de especies } & 6 & 4 & 5 & 4 & 4\end{array}$

Caract. de comunidad y unidades superiores

$\begin{array}{lllllll}\text { Ceratophyllum demersum } & 5.5 & 4.4 & 4.4 & 3.3 & 3.3\end{array}$

Compañeras

$\begin{array}{llllll}\text { Myriophyllum spicatum } & 1.1 & 2.2 & 2.2 & \text {. } & 3.3\end{array}$

$\begin{array}{llllll}\text { Phragmites australis } & 1.2 & \text {. } & 1.2 & \text {. } & 1.2\end{array}$

$\begin{array}{llllll}\text { Sparganium erectum } & \text {. } & 1.1 & +.2 & & +.2\end{array}$

$\begin{array}{lllll}\text { Nasturtium officinale } \quad . & + & +2 & 1.2\end{array}$.

$\begin{array}{lllll}\text { Typha dominguensis } & +.2 & +.2 & .\end{array}$.

Además: En 1.- Juncus effusus, 1.1; Carex otrubae, 1.1. En 4.- Lemna gibba, 1.1; Iris pseudacorus, +.2 .

Localidades: 1.- Manantial de Ojo Oscuro (VF4697). 2.- Acequia de la Madre Maestra (VF4697). 3.- Cenefa de los Ojos (VF4697). 4.- Acequia de la Madre Maestra (VF4696). 5.Turbera de Aguadero (VF4697).

\section{III.- $C H A R E T E A$ Fukarek 1961}

Comunidades constituidas principalmente por carófitos (rizófitos) que se desarrollan tanto en estanques y lagunas de aguas frescas, oligo-eútrofas, como en aguas oligo-mesohalinas y salobres. Representada en la Depresión de Padul por el orden Charetalia y la alianza Charion fragilis, que engloba las comunidades de carófitos de aguas frescas no salinas. 


\section{3.- Charetum vulgaris Corillion 1957}

Comunidad caracterizada por la presencia de Chara vulgaris (tabla 3), que coloniza fondos poco profundos de lagunas endorreicas y acequias de aguas dulces, tranquilas, y generalmente poco eutrofizadas, donde forma un auténtico cesped sumergido en ocasiones por debajo de otras comunidades de plantas acuáticas. Principalmente se desarrolla sobre fondos arenosos, pero también aparece sobre sustratos finos, fangosos. Ampliamente extendida por Andalucía (Asensi y Nieto, 1981), encuentra una buena representación en la zona.

\section{TABLA 3}

Charetum vulgaris Corillion 1957

(Charion fragilis, Charetalia, Charetea)

\begin{tabular}{lrrr}
\hline № de inventario & 1 & 2 & 3 \\
Area $\left(\mathrm{m}^{2}\right)$ & 5 & 9 & 3 \\
Cobertura (\%) & 90 & 90 & 70 \\
№ de especies & 6 & 3 & 4 \\
& & & \\
Caract. de asociación y unidades superiores & \\
Chara vulgaris & 4.4 & 3.4 & 3.3 \\
& & & \\
Compañeras & & & \\
Scirpus setaceus & +.2 &. & +.2 \\
Myriophyllum spicatum & $\cdot$ & 3.3 & $\cdot$ \\
Chiloscyphus polyanthos & $\cdot$ & 2.2 & $\cdot$ \\
Apium nodiflorum & $\cdot$ & $\cdot$ & 2.2 \\
Zannichellia pedunculata & 1.2 & $\cdot$ & $\cdot$ \\
Juncus articulatus & 1.1 & $\cdot$ & $\cdot$ \\
Lythrum salicaria & +.2 & $\cdot$ & $\cdot$ \\
Nasturtium officinale & +.2 & $\cdot$ & $\cdot$ \\
Agrostis stolonifera &. & $\cdot$ & +.2
\end{tabular}

Localidades: 1.- Nacimiento de la Venta(VF4496). 2.- Acequia de Agía(VF4495). 3.- Fuente de la Esperanza (VF4496).

IV.- POTAMETEA R.Tx. \& Preising 1942

Vegetación cormofítica arraigada de aguas dulces, constituida fundamentalmente por comunidades de elodeidos, ninfeidos, miriofílidos y batráchidos. Aparecen representados los órdenes Magnopotametalia, Parvopotametalia y LuronioPotametalia. 


\section{A) MAGNOPOTAMETALIA Den Hartog \& Segal 1964}

Comunidades de largos elodeidos y ninfeidos de aguas profundas eútrofas y mesoeútrofas. Representado por la alianza Magnopotamion que agrupa las comunidades de largas especies de Potamogeton y otros elodeidos.

\section{4.- Comunidad de Potamogeton pectinatus L.}

Con un desarrollo primaveral-estival, aparece una comunidad oligofítica muy densa de Potamogeton pectinatus (tabla 4), que presenta un crecimiento en forma de largos tallos que con frecuencia superan los $2 \mathrm{~m}$. Se presenta en canales de aguas eútrofas de corriente media a elevada, con una profundidad que varía de 1 a $3 \mathrm{~m}$. El tratamiento sintaxonómico de las comunidades de Potamogeton pectinatus, ha sido controvertido según algunos autores. Por nuestra parte consideramos acertada su inclusión en el orden Magnopotametalia, como indica Cirujano (1981).

\section{TABLA 4}

Comunidad de Potamogeton pectinatus L.

(Magnopotamion, Magnopotametalia, Potametea)

$\begin{array}{lccc}\text { № de inventario } & 1 & 2 & 3 \\ \text { Area }\left(\mathrm{m}^{2}\right) & 8 & 5 & 4 \\ \text { Cobertura }(\%) & 100 & 90 & 90 \\ \text { Profundidad }(\mathrm{m}) & 1,5 & 2 & 2,5 \\ \text { № de especies } & 5 & 4 & 3\end{array}$

Caract. de comunidad y unidades superiores

$\begin{array}{lccc}\text { Potamogeton pectinatus } & 5.5 & 5.5 & 5.5 \\ \text { Myriophyllum spicatum } & 1.1 & . & . \\ \text { Compañeras } & & & \\ \text { Veronica anagallis-aquatica } & +.2 & +.2 & +.2 \\ \text { Chara vulgaris } & +.2 & . & . \\ \text { Chiloscyphus polyanthos } & +.2 & . & . \\ \text { Cyperus longus } & . & +.2 & . \\ \text { Iris pseudacorus } & . & +.2 & . \\ \text { Nasturtium officinale } & . & . & +.2\end{array}$

Localidades: 1.- Acequia de Agía (VF4595). 2.- Acequia del Ventano (VF4596). 3.- Acequia de la Madrecilla de Quiti (VF4696)

B) PARVOPOTAMETALIA Den Hartog \& Segal 1964

Agrupa las comunidades de pequeños elodeidos de aguas estancadas y poco 
profundas, así como las de batráchidos de aguas temporales. Hemos localizado en la Depresión de Padul una comunidad de pequeños elodeidos incluible en la alianza Parvopotamion.

5.- Comunidad de Zannichellia pedunculata Reichenb.

Comunidad de desarrollo primaveral-estival, en la que es constante la presencia de Zannichellia pedunculata, acompañada normalmente por Myriophyllum spicatum (tabla 5). Se presenta en acequias y charcas de aguas eútrofas, estancadas o de corriente débil, cuyo nivel de agua disminuye de forma considerable en el verano.

\section{TABLA 5}

Comunidad de Zannichellia pedunculata Reichenb.

(Parvopotamion, Parvopotametalia, Potametea)

№ de inventario
Area $\left(\mathrm{m}^{2}\right)$
Cobertura $(\%)$
Profundidad $(\mathrm{m})$
№ de especies

Caract. de comunidad y unidades superiores

Zannichellia pedunculata

Myriophyllum spicatum

Potamogeton pectinatus

Compañeras

Nasturtium officinale

Apium nodiflorum

Agrostis stolonifera

Chara vulgaris

Scirpus setaceus

Glyceria declinata

Cyperus longus

Juncus articulatus

$\begin{array}{cccc}1 & 2 & 3 & 4 \\ 2 & 2 & 4 & 10 \\ 100 & 100 & 90 & 100 \\ 0,5 & 0,5 & 0,5 & 1 \\ 5 & 6 & 5 & 5\end{array}$

$\begin{array}{cccc}5.5 & 4.4 & 3.4 & 2.3 \\ 1.1 & 3.3 & . & 3.4 \\ . & . & . & 2.2\end{array}$

$\begin{array}{llll}+.2 & 1.1 & +.2 & 1.2\end{array}$

$\begin{array}{llll}1.1 & +.2\end{array}$

$+.2+.2$

. $\quad 2.2$

$\cdot \quad 1.1$

+.2 ..

$+.2$

Localidades: 1 y 2.- Fuente de la Salud (VF4597). 3.- Fuente de la Esperanza (VF4496). 4.Acequia de los Quinientos (VF4596).

C) LURONIO-POTAMETALIA Den Hartog \& Segal 1964

Incluye las comunidades de elodeidos y pequeños ninfeidos de aguas oligomeso y distrofas poco profundas. Se encuentra de manera muy puntual y fragmentaria en la Depresión de Padul, representado por la alianza Potamion graminei. 
6.- Comunidad de Potamogeton coloratus Hornem.

Se presenta en aguas claras mesoeútrofas algo alcalinas, de corriente media, una comunidad de Potamogeton coloratus acompañado de algunos rizófitos (tabla 6), que siguiendo a GEHU \& BOURNIQUE (1987) es incluible en la alianza Potamion graminei. Se trata de la única cita reciente para el SE peninsular, lo que realza el valor de esta comunidad, teniendo en cuenta la fragilidad del ecosistema que ocupa.

\section{TABLA 6}

Comunidad de Potamogeton coloratus Hornem. (Potamion graminei, Luronio-Potametalia, Potametea)

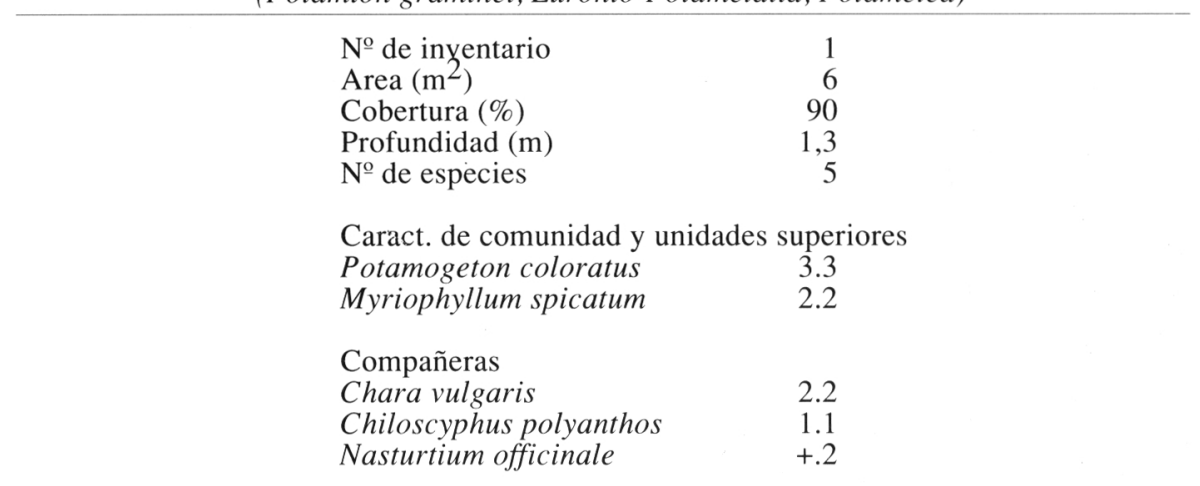

Localidad: 1.- Acequia de Agía (VF4495).

V.- ISOETO-NANOJUNCETEA Br.B1. \& R.Tx. 1943

Vegetación terofítica y pionera, desarrollada sobre suelos periódicamente encharcados o cubiertos por aguas dulces o salobres, que presentan en general un corto periodo de vida vegetativa. En la Depresión de Padul hemos reconocido un orden, Isoetalia que comprende las comunidades de floración preestival, mediterráneas y occidental-europeas de carácter subtermófilo. Se encuentra representado por la alianza Cicendion, que agrupa las asociaciones fugaces de pequeña talla, desarrolladas en zonas encharcadas o cubiertas algunos meses por aguas superficiales.

7.- Comunidad de Juncus bufonius L.

Aparece de forma fragmentaria y empobrecida en especies características, lo que unido a la fugacidad de su periodo vegetativo nos ha impedido su encuadre 
sintaxonómico a nivel de asociación. Por ello nos hemos limitado a seleccionar 6 inventarios (tabla 7) que consideramos representativos de una comunidad de Juncus bufonius, acompañado de otros terófitos de apetencias halonitrófilas y algún hemicriptófito.

Se trata de prados terofíticos de cobertura media a densa, desarrollados en áreas próximas a lagunas y acequias de riego, encharcadas de forma periódica, y que presentan acúmulo de sales en superficie cuando se desecan. Su inclusión en la alianza Cicendion se basa en criterios ecológicos y fenológicos, y no estrictamente en base a su composición florística.

\section{TABLA 7}

Comunidad de Juncus bufonius L.

(Cicendion, Isoetalia, Isoeto-Nanojuncetea)

№ de inventario

Area $\left(\mathrm{m}^{2}\right)$

Cobertura $(\%)$

№ de especies

$\begin{array}{cccccc}1 & 2 & 3 & 4 & 5 & 6 \\ 9 & 4 & 6 & 9 & 20 & 3 \\ 90 & 90 & 90 & 80 & 80 & 80 \\ 7 & 9 & 9 & 9 & 9 & 8\end{array}$

Caract. de comunidad

Juncus bufonius

$5.5 \quad 5.5$

4.4

4.4

3.4

3.3

Compañeras

Polypogon monspeliensis

$\begin{array}{llllll}1.2 & 2.2 & 3.3 & 2.2 & 3.3 & 2.3\end{array}$

Hymenolobus procumbens

$\begin{array}{llllll}2.2 & . & 1.1 & 1.1 & +.2 & 1.1\end{array}$

Ranunculus muricatus

$+.2+.2+.2$

Poa annua

Cynodon dactylon

Anagallis arvensis

Rumex crispus

Trifolium repens

Spergularia marina

Alopecurus arundinaceus

. 1.22

$+.2+.2$

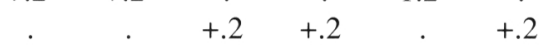

+.2 . $\quad+.2+.2$.

$\begin{array}{lllll}. & 1.2 & +.2 & . & .\end{array}$

$\begin{array}{ccccc}1.1 & \cdot & \cdot & \cdot & +.2 \\ . & \cdot & +.2 & \cdot & .\end{array}$

Además: En 2.- Calystegia sepium, 1.1; Veronica anagallis-aquatica, +.2 ; Ranunculus trilobus, 1.1; Plantago media, +.2; Scirpus maritimus, +.2. En 3.- Nasturtium officinale, +.2. En 4.Scirpus holoschoenus, +.2. En 5.- Scirpus tabernaemontani, +.2; Typha dominguensis, +.2; Polygonum lapathifolium, +.2. En 6.-Equisetum ramosissimum, 1.1; Sonchus tenerrimus, +. 2.

Localidades: 1 y 5.- Berrazal (VF4597). 2.- Turbera de Agía (VF4495). 3 y 4.- Fuente de la Salud (VF4497). 6.- Acequia de la Madre Maestra (VF4597). 
VI.- PHRAGMITETEA R.Tx. \& Preising 1942

Vegetación hidrófila enraizada, constituida por grandes helófitos o hierbas jugosas helofíticas, propia de los bordes de cursos de agua, lagunas, zonas pantanosas y otras estaciones hidrófilas permanentes. En el territorio hemos reconocido el orden Phragmitetalia, que agrupa las comunidades propias de aguas poco o nada salinas y representado por tres alianzas: Phragmition, Glycerio-Sparganion y Magnocaricion elatae.

a) Phragmition australis W.Koch 1926

Comprende las asociaciones de carrizales y espadañares, de altura considerable, desarrolladas en suelos profundos; las comunidades de esta alianza son las que presentan una mayor exigencia de agua dentro de la clase Phragmitetea. 2 asociaciones.

\section{8.- Typho-Scirpetum tabernaemontani Br.Bl. \& O.Bolós 1957}

Carrizales, juncales o espadañares, generalmente pobres florísticamente, cuya fisionomía suele ser variable en función de la dominancia de alguna de las especies características. Se desarrolla sobre suelos compactos, ricos en materia orgánica, generalmente con un horizonte negro de potencia variable, que se presentan encharcados la mayor parte del año (tabla 8).

Esta asociación sería la más pobre florísticamente y la más meridional de la alianza. Próxima al Scirpo-Phragmitetum mediterraneum R.Tx. \& Preising 1942, se caracteriza por la ausencia total de Scirpus lacustris que es reemplazado por Scirpus tabernaemontani, y por la abundancia de Typha dominguensis frente a otras especies del género; también es de destacar la relativa frecuencia de Scirpus maritimus en la asociación. Ampliamente representada en la Depresión de Padul, donde ocupa grandes extensiones.

\section{9.- Scirpetum maritimi (Christiansen 1934) R.Tx. 1937}

Asociación en la que suele dominar Scirpus maritimus, que se desarrolla en suelos principalmente arcillosos, cubiertos por aguas profundas la mayor parte del año (tabla 9). No es una asociación claramente halófila, aunque puede soportar cierta salinidad estacional. En el territorio se encuentra extendida en suelos encharcados de los bordes de lagunas y charcas, donde al perderse la capa de agua en la época estival se presenta cierta salinidad superficial. Esta comunidad sólo ha sido citada en Andalucía por Rivas-Martínez et al. (1980) para Doñana.

\section{b) Glycerio-Sparganion Br.B1. \& Sissing in Boer 1942}

Representa las asociaciones de helófitos megafórbicos o graminoides propios de cauces poco profundos de aguas más o menos rápidas y someras. 2 asociaciones. 


\section{TABLA 8}

Typho-Scirpetum tabernaemontani Br.B1. \& O.Bolós 1957 (Phragmition, Phragmitetalia, Phragmitetea)

\begin{tabular}{lrrrrrrrr}
\hline № de inventario & 1 & 2 & 3 & 4 & 5 & 6 & 7 & 8 \\
Area $\left(\mathrm{m}^{2}\right)$ & 100 & 100 & 100 & 100 & 50 & 100 & 200 & 50 \\
Cobertura $(\%)$ & 100 & 100 & 100 & 100 & 90 & 100 & 100 & 100 \\
№ de especies & 7 & 15 & 8 & 11 & 10 & 12 & 10 & 16
\end{tabular}

Caract. de asociación y unidades superiores

\begin{tabular}{|c|c|c|c|c|c|c|c|c|}
\hline Phragmites australis & 4.4 & 1.1 & +.2 & . & 2.2 & 4.4 & 5.5 & 3.4 \\
\hline Typha dominguensis & 3.3 & 3.3 & 2.2 & 3.4 & 3.4 & 1.2 & . & 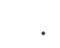 \\
\hline Scirpus tabernaemontani & 2.2 & 3.4 & 4.4 & 3.4 & . & . & . & \\
\hline Epilobium hirsutum & . & +.2 & . & 1.2 & . & . & 2.2 & 1.1 \\
\hline Scirpus maritimus & . & 1.1 & 1.1 & 1.1 & . & . & $\cdot$ & \\
\hline Lythrum salicaria & . & . & . & . & . & . & 2.2 & 1.1 \\
\hline Sparganium erectum & 1.1 & . & . & . & 1.2 & . & . & \\
\hline Althaea officinalis & . & . & . & . & $\cdot$ & 1.1 & . & +.2 \\
\hline Lycopus europaeus & . & . & . & . & . & +.2 & . & +.2 \\
\hline Veronica anagallis-aquatica & . & . & . & . & +.2 & . & +.2 & \\
\hline Nasturtium officinale & . & . & . & . & . & . & 1.1 & \\
\hline \multicolumn{9}{|l|}{ Compañeras } \\
\hline Scirpus holoschoenus & +.2 & 2.2 & 1.2 & 1.2 & . & +.2 & 1.1 & 1.1 \\
\hline Sonchus aquatilis & . & $\cdot$ & $\cdot$ & 1.1 & +.2 & 1.1 & +.2 & +.2 \\
\hline Cynodon dactylon & 1.1 & 1.2 & 2.2 & 2.2 & . & . & . & \\
\hline Convolvulus arvensis & $\cdot$ & . & 1.1 & $\cdot$ & +.2 & 1.1 & . & +.2 \\
\hline Trifolium pratense & . & 1.1 & . & . & +.2 & +.2 & . & +.2 \\
\hline Juncus subulatus & +.2 & 1.2 & 2.2 & . & . & . & . & . \\
\hline Polypogon monspeliensis & . & +.2 & . & 1.2 & . & . & . & +.2 \\
\hline Rumex pulcher & . & +.2 & . & +.2 & . & 1.1 & . & . \\
\hline Dorycnium rectum & . & . & . & . & 1.2 & . & 1.2 & . \\
\hline Festuca arundinacea & . & 1.1 & . & . & 1.1 & . & . & . \\
\hline Calystegia sepium & . & 1.1 & . & . & . & . & . & 1.1 \\
\hline Cirsium micranthum & . & . & . & . & . & . & 1.1 & 1.1 \\
\hline Mentha suaveolens & . & . & . & . & . & +.2 & . & +.2 \\
\hline
\end{tabular}

Además: En 2.- Ranunculus repens, 1.1; Holcus lanatus, +.2. En 4.- Lythrum junceum, 1.1; Cyperus longus, +.2. En 5.- Brachypodium phoenicoides, 1.1. En 6.- Potentilla reptans, 1.1; Galium aparine, +.2. En 7.- Scutellaria galericulata, +.2. En 8.- Euphorbia pubescens, +.2 ; Scrophularia auriculata, +.2 ; Thalictrum speciosum, +.2 .

Localidades: 1.- Berrazal (VF4597). 2 y 4.- Cenefa de los Ojos (VF4697). 3.- Cenefa de Enmedio (VF4696). 5.- Acequia de Los Quinientos (VF4596). 6.- Acequia del Brazal (VF4596). 7.- Manantial de Ojo Oscuro (VF4697). 8.- Acequia de Río Viejo (VF4595). 
10.- Helosciadetum nodiflori $\mathrm{Br} . \mathrm{B} 1.1931$

Asociación constituida por hierbas jugosas y tiernas, siempre verdes, dominadas por Apium nodiflorum y Nasturtium officinale, que coloniza los bordes de cursos de agua dulce más o menos eutrofizados, poco profundos y generalmente permanentes, sobre sustratos arcillosos, limosos o, incluso, fangosos (tabla 10). Se presenta ampliamente extendida en la mayor parte de las acequias y arroyos de la zona.

\section{TABLA 9}

Scirpetum maritimi (Christiansen 1934) R.Tx. 1937

(Phragmition, Phragmitetalia, Phragmitetea)

№ de inventario

Area $\left(\mathrm{m}^{2}\right)$

Cobertura $(\%)$

№ de especies

$\begin{array}{rccc}1 & 2 & 3 & 4 \\ 12 & 12 & 8 & 20 \\ 90 & 90 & 80 & 90 \\ 8 & 9 & 7 & 8\end{array}$

Caract. de asociación y unidades superiores

Scirpus maritimus

Phragmites australis

4.4

$\begin{array}{lll}4.4 & 4.4 \quad 3.4\end{array}$

Scirpus tabernaemontani

$+.2+.2 \quad 1.1$

Lythrum salicaria

Lythrum salicaria

.

1.2

Compañeras

Cynodon dactylon

Festuca arundinacea

$\begin{array}{cccc}1.1 & 1.2 & 1.2 & 1.2 \\ 1.1 & 2.2 & . & 1.1 \\ . & +.2 & 1.2 & 1.2 \\ 1.2 & . & 2.2 & . \\ 2.2 & . & 1.1 & . \\ +.2 & +.2 & . & . \\ . & +.2 & . & +.2 \\ . & +.2 & . & +.2\end{array}$

Además: En 1.-Spergularia marina, 2.2; Blackstonia perfoliata,+.2. En 2.-Trifolium pratense, 1.2. En 3.- Calystegia sepium, +.2. En 4.- Lotus corniculatus, +.2; Trifolium repens, +.2.

Localidades: 1.- Berrazal (VF4597). 2.- Acequia de la Madre Blanca (VF4696). 3.- Turbera de Agía (VF4595). 4.- Cenefa de Enmedio (VF4696). 
TABLA 10

Helosciadetum nodiflori Br.Bl. 1931

(Glycerio-Sparganion, Phragmitetalia, Phragmitetea)

$\begin{array}{lcccc}\text { № de inventario } & 1 & 2 & 3 & 4 \\ \text { Area }\left(\mathrm{m}^{2}\right) & 4 & 5 & 2 & 3 \\ \text { Cobertura }(\%) & 100 & 100 & 90 & 100 \\ \text { № de especies } & 8 & 8 & 14 & 8\end{array}$

Caract. de asociación y unidades superiores

Nasturtium officinale

$\begin{array}{llll}5.5 & 3.3 & 3.3 & 4.4 \\ 1.1 & 4.4 & 3.3 & 1.2 \\ . & . & 1.2 & 1.1 \\ 1.1 & . & . & +.2 \\ . & . & . & 1.2 \\ . & . & . & +.2\end{array}$

Compañeras

Sonchus aquatilis

Festuca arundinacea

Mentha suaveolens

Polygonum lapathifolium

Euphorbia pubescens

Scrophularia auriculata

Rumex pulcher

$\begin{array}{cccc}+.2 & 1.1 & +.2 & . \\ 1.2 & 1.1 & . & . \\ \cdot & 1.1 & 1.1 & . \\ . & . & 1.2 & +.2 \\ . & +.2 & 1.1 & . \\ . & 1.1 & +.2 & . \\ +.2 & . & +.2 & .\end{array}$

Además: En 1.- Scirpus holoschoenus, 1.2; Carex chaetophylla, 1.2. En 2.- Tetragonolobus maritimus, 1.2. En 3.- Cyperus longus, 1.2; Cynodon dactylon, $+.2 ;$ Equisetum ramosissimum, +.2 ; Atriplex hastata, +.2 ; Myriophyllum spicatum, +.2 . En 4.- Calystegia sepium, +.2.

Localidades: 1.- Fuente de la Higuera (VF4597). 2.- Acequia de Los Quinientos (VF4596). 3.- Fuente de la Salud (VF4497). 4.- Cenefa de los Ojos (VF4697).

\section{1.- Junco subnodulosi-Sparganietum erecti Martínez Parras y Peinado 1983}

Comunidad helofítica dominada por Sparganium erectum, normalmente acompañado de otros helófitos, juncos, etc.; se desarrolla en los bordes de lagunas, canales y acequias o formando un denso mosaico con los espadañares y carrizales del Typho-Scirpetum tabernaemontani, sobre suelos compactos, generalmente ricos en materia orgánica y que permanecen encharcados gran parte del año. Asociación descrita para la Depresión de Padul por Martínez-Parras y Peinado (1983), donde se encuentra muy bien representada. 


\section{TABLA 11}

Junco subnodulosi-Sparganietum erecti Martínez Parras y Peinado 1983 (Glycerio-Sparganion, Phragmitetalia, Phragmitetea)

$\begin{array}{lccccc}\text { № de inventario } & 1 & 2 & 3 & 4 & 5 \\ \text { Area }\left(\mathrm{m}^{2}\right) & 10 & 60 & 100 & 10 & 10 \\ \text { Cobertura }(\%) & 100 & 100 & 80 & 100 & 100 \\ \text { № de especies } & 4 & 6 & 6 & 8 & 7\end{array}$

Caract. de asociación y unidades superiores

\begin{tabular}{|c|c|c|c|c|c|}
\hline Sparganium erectum & 5.5 & 5.5 & 3.4 & 5.5 & 5.5 \\
\hline Juncus subnodulosus & . & 3.3 & 2.2 & . & +.2 \\
\hline Typha dominguensis & +.2 & 1.2 & 1.2 & . & \\
\hline Cyperus longus & 4.4 & . & . & . & 2.2 \\
\hline Lythrum salicaria & . & . & 2.3 & 1.2 & . \\
\hline Epilobium hirsutum & . & 1.2 & 1.2 & . & . \\
\hline Scirpus tabernaemontani & . & 1.2 & . & . & 1.1 \\
\hline Phragmites australis & +.2 & 1.2 & . & . & . \\
\hline Veronica anagallis-aquatica & . & . & . & . & + \\
\hline \multicolumn{6}{|l|}{ Compañeras } \\
\hline Festuca arundinacea & . & . & . & 3.3 & . \\
\hline Scirpus holoschoenus & . & . & 1.2 & . & . \\
\hline Euphorbia pubescens & . & . & . & 1.1 & . \\
\hline Tetragonolobus maritimus & . & . & . & 1.1 & . \\
\hline Scrophularia auriculata & . & . & . & 1.1 & . \\
\hline Trifolium repens & . & . & . & +.2 & . \\
\hline Senecio vulgaris & . & . & . & +.2 & . \\
\hline Juncus effusus & . & . & . & . & +.2 \\
\hline Rumex crispus & . & . & . & . & + \\
\hline
\end{tabular}

Localidades: 1.- Berrazal (VF4597). 2.- Acequia de la Madre Maestra (VF4697). 3.- Cenefa de los Ojos (VF4697). 4.- Fuente de la Esperanza (VF4597). 5.- Depresión de Padul (Inv.4, Tab.5 de Martínez Parras y Peinado 1983:324. Sintypus de la asociación).

c) Magnocaricion elatae W.Koch 1926

Incluye las asociaciones de grandes helófitos amacollados graminoides, sometidas a encharcamiento variable.

12.- Cladio marisci-Caricetum hispidae O.Bolós 1967

Asociación de elevada cobertura y mediana talla, generalmente presidida por Carex hispida, propia de suelos turbosos húmedos, poco permeables y encharcados 
gran parte del año. Descrita para la provincia corológica Valenciano-CatalanoProvenzal, su presencia en la Bética ha sido puesta de manifiesto recientemente por GOMEZ MERCADO (1989:86) para el Valle del Guadalquivir. Nosotros confirmamos su presencia ampliándola al sector Malacitano-Almijarense, si bien, nuestros inventarios resultan pobres florísticamente.

\section{TABLA 12}

Cladio marisci-Caricetum hispidae O.Bolós 1967

(Magnocaricion, Phragmitetalia, Phragmitetea)

\begin{tabular}{lcc}
\hline № de inventario & 1 & 2 \\
Area $\left(\mathrm{m}^{2}\right)$ & 20 & 10 \\
Cobertura (\%) & 100 & 100 \\
№ de especies & 10 & 13 \\
& & \\
Caract. de asociación y unidades superiores & \\
Carex hispida & 5.5 & 4.4 \\
Phragmites australis & +.2 & $\cdot$ \\
Juncus subnodulosus & +.2 & $\cdot$ \\
Veronica anagallis-aquatica & $\cdot$ & +.2 \\
& & \\
Compañeras & & \\
Convolvulus arvensis & 1.2 & 2.2 \\
Festuca arundinacea & 1.2 & 2.2 \\
Potentilla reptans & 1.1 & 1.1 \\
Elymus repens & 1.1 & 1.1
\end{tabular}

Además: En 1.- Scrophularia auriculata, 1.2; Euphorbia pubescens, 1.1; Trifolium repens, +.2. En 2.- Ranunculus repens, 1.1; Trifolium pratense, 1.1; Rumex pulcher, 1.1; Equisetum ramosissimum, 1.1; Mentha rotundifolia, +.2 ; Sonchus aquatilis, +.2 ; Scirpus holoschoenus, +.2 .

Localidades: 1.- Acequia del Ventano (VF4596). 2.- Acequia de Río Viejo (VF4495).

\section{ESQUEMA SINTAXONÓMICO}

I.- LEMNETEA MINORIS W.Koch \& R.Tx. in R.Tx. 1955

* LEMNETALIA MINORIS W.Koch \& R.Tx. in R.Tx. 1955

+ Lemnion gibbae R.Tx. \& Schwabe in R.Tx. 1974

1.- Lemnetum gibbae (W.Koch 1954) Miyawaki \& R.Tx. 1960

II.- CERATOPHYLLETEA Den Hartog \& Segal 1964

* CERATOPHYLlETALIA Den Hartog \& Segal 1964 
+ Ceratophyllion Den Hartog \& Segal 1964

2.- Comunidad de Ceratophyllum demersum L.

III.- CHARETEA Fukarek 1961

* CHARETALIA Sauer 1937

+ Charion fragilis Krausch 1964

3.- Charetum vulgaris Corillion 1957

IV.- POTAMETEA R.Tx. \& Preising 1942

* MAGNOPOTAMETALIA Den Hartog \& Segal 1964

+ Magnopotamion Vollmar 1947

4.- Comunidad de Potamogeton pectinatus L.

* PARVOPOTAMETALiA Den Hartog \& Segal 1964

+ Parvopotamion Vollmar 1947

5.- $\quad$ Comunidad de Zannichellia pedunculata Reichenb.

* LURONIO-POTAMETALIA Den Hartog \& Segal 1964

+ Potamion graminei (Den Hartog \& Segal 1964) Westhoff \& Den Held 1969

6.- Comunidad de Potamogeton coloratus Hornem.

V.- ISOETO-NANOJUNCETEA Br.Bl. \& R.Tx. 1943

* ISOETALIA Br.B1. 1931 em. Rivas Goday 1970

+ Cicendion (Rivas Goday (1961) 1964) Br.Bl. 1967

7.- Comunidad de Juncus bufonius L.

VI.- PHRAGMITETEA R.Tx. \& Preising 1942

* PHRAGMITETALIA W.Koch 1926

+ Phragmition australis W.Koch 1926 em. Br.Bl. 1931

8.- Typho-Scirpetum tabernaemontani Br.Bl. \& O.Bolós 1957

9.- $\quad$ Scirpetum maritimi (Christiansen 1934) R.Tx. 1937

+ Glycerio-Sparganion Br.Bl. \& Sissingh in Boer 1942

10.- Helosciadetum nodiflori Br.Bl. 1931

11.- Junco subnodulosi-Sparganietum erecti Martínez Parras y Peinado 1983

+ Magnocaricion W.Koch 1926

12.- Cladio marisci-Caricetum hispidae O.Bolós 1967

AGRADECIMIENTOS. A J.M. Pizarro y J.A. Molina por la comprobación de las especies de hidrófitos así como por sus observaciones sobre este tipo de comunidades, y a J.A. Gil por la determinación de la hepática Chiloscyphus polyanthos (L.) Dum.

\section{BIBLIOGRAFÍA}

ASENSI,A. y J.M. NIETO CALDERA-1981- Vegetación acuática, halófila y halonitrófila de la provincia de Málaga. Trab. y Monograf.Dep.Bot.Málaga, 2:105-122.

CIRUJANO, S.-1981- Las lagunas manchegas y su vegetación. I. Anales Jard.Bot.Madrid, 37(1):155-192. 
DEN HARTOG, C. \& S. SEGAL -1964- A new classification of the water-plant communities. Acta Botánica Neerlandica, 13:367-393.

ELÍAS-CASTILLO, F. y L. RUIZ-BELTRAN -1977-Agroclimatología de España.Cuadernos del I.N.I.A., 7.

GEHU, J.M. \& C.P. BOURNIQUE.-1987-Schéma synsystematique des associations aquatiques et amphibies du nord de la France. Secr.Publ.Univ. La Laguna, no22: 321-326. Santa Cruz de Tenerife.

GÓMEZ-MERCADO, F.-1989- Cartografía y estudio de la vegetación de la Sierra de Cazorla. Tesis Doctoral (inéd.). Univ. Granada.

MARTÍNEZ-PARRAS, J.M. y M. PEINADO-1983- Estudio botánico de los ecosistemas de la Depresión de Padul (Granada). Collect.Bot., 14:317-326. Barcelona.

RIVAS-MARTÍNEZ, S.-1987- Mapa y Memoria de las series de vegetación de España. Escala 1:400.000. Serv.Publ.Min.Agric.Pesc. y Alim. ICONA. Madrid.

RIVAS-MARTÍNEz, S., A. ASENSI, J. MOLERO y F. VALLE-1987-Tipología biogeográfica de Andalucía. I Jornadas de Taxonomía Vegetal (Multicopia). Sevilla.

RIVAS-MARTINEZ, S., M. COSTA, S. CASTROVIEJO y B. VALDÉS -1980- Vegetación de Doñana (Huelva, España). Lazaroa, 2:5-190. Madrid.

(Aceptado para su publicación en Enero de 1991)

Dirección de los autores: Departamento de Biología Vegetal (Botánica). Universidad de Granada. 18071 Granada. 